Series A

I. MATHEMATICA

433

\title{
TWO REMARKS CONCERNING DIRICHLET'S L-FUNCTIONS AND THE CLASS NUMBER OF THE CYCLOTOMIC FIELD
}

BY

TIMO LEPISTÖ

H E L S N K I 1968

S U O M A L A N EN TIEDEAKATEMIA

doi:10.5186/aasfm.1969.433 
Communicated 10 May 1968 by P. J. Myrberg and K. InkerI. 


\section{TWO REMARKS CONCERNING DIRICHLET'S $L$-FUNCTIONS AND THE CLASS NUMBER OF THE CYCLOTOMIC FIELD}

1. In the following we denote by $K$ an algebraic number field of degree $n$ over the field $P$ of rational numbers. In addition let $d$ denote the discriminant, $R$ the regulator and $h$ the number of classes of ideals of $K$. BraUer (cf. [3], p. 745) has proved the following')

Theorem 1. If $K$ ranges over a sequence of fields normal over $P$ for which $n / \log |d| \rightarrow 0$, then

$$
\log (R h) \sim \log \sqrt{|\bar{d}|} .
$$

This theorem treats very general cases. If we restrict ourselves to the cyclotomic field $K=P(\zeta)$, where $\zeta$ is a primitive $p$ th $(p$ is an odd prime.) root of unity, we have

Theorem 2. For any positive $\varepsilon$,

$$
2 p^{\frac{1}{2} p}(2 \pi)^{\frac{1}{2}(1-p)} c(\varepsilon) p^{-\varepsilon}<R h<2 p^{\frac{1}{2} p}(2 \pi)^{\frac{1}{2}(1-p)} \log ^{c} p .
$$

where $c$ and $c(\varepsilon)$ denote respectively an absolute positive constant and $a$ positive constant depending on parameter $\varepsilon$ alone.

This result introduced by Tatuzawa (cf. [8], p. 111) gives in this special case a sharper estimation than the one of theorem 1.

In this paper we consider the cyclotomic field $K=P(\zeta)$, where $\zeta$ is a primitive $m$ th root of unity. We suppose that the natural number $m$ is $>1$ and in addition we exclude those even values of $m$, which are not divisible by 4 . This restriction is not essential because both the primitive $m$ th and $(m / 2)$ th roots of unity generate the same field if $m$ has some excluded value. We are able to extend theorem 2 and prove

Theorem 3. If $c$ and $c(\varepsilon)$ are defined as above then

$$
c(\varepsilon) m^{-\varepsilon}<R h / G<\exp (c(\log \log m+\omega(m))),
$$

1) The result of theorem 1 is an improvement of BRAUER's earlier result (cf. [2], p. 243). This estimation cannot, however, be applied in the case of the cyclotomic field, because it treats only the cases, where $d$ tends to infinity in such a way that the degree of the corresponding fields is fixed. It should be noted that Vinogradov (cf. [9], p. 562) has presented estimations, which complete the result of BraUER, but which cannot be applied here, because they also presuppose that the degree of the fields under examination is fixed. 
where

$$
G=(2 \pi)^{-\frac{1}{2} q(m)} w \sqrt{|d|}
$$

(As usual, $\varphi$ denotes Euler's function.). Here

$$
w=\left\{\begin{array}{c}
2 m \text { if } 2+m, \\
m \text { if } 2 \mid m
\end{array}\right.
$$

is the number of roots of 1 contained in $P(\zeta)$.

It should be mentioned that it is possible to apply theorem 1 in the case of the cyclotomic field. It is known that this field is normal over $P$ and in addition we have

$$
\log |d| \geqq 1 / 2 \varphi(m) \log m
$$

(cf. [6], p. 27 and [5], p. 508). This yields

$$
0<n / \log |d| \leqq 2 / \log m \rightarrow 0,
$$

when $m$ tends to infinity. It is, however, easy to see that in the case of the cyclotomic field the result of theorem 3 is sharper than the one of theorem 1 .

2. In addition we consider the so-called Dirichlet's $L$-functions

$$
L(s, \chi)=\sum_{n=1}^{\infty} \chi(n) n^{-s}
$$

Here $\chi(n)$ denotes a character $(\bmod k)$, where $k$ is an arbitrary natural number and $s$ is a real variable. For these functions we have (cf. [6], p. 31)

Theorem 4. Let $\varepsilon$ be an arbitrary positive number, $\delta$ a positive number $<1 / 2$ and

$$
\tau=\tau(k)=\left\{\begin{array}{l}
\omega(k) \text { if } 2+k, \\
\omega(k)+1 \text { if } 2 \mid k,
\end{array}\right.
$$

where $\omega(k)$ denotes the number of different prime factors of $k$. If the extended Riemann hypothesis is true, there exists for every given pair $s$ and $k(\geqq 3)$, where

$$
s \geqq(\tau+\delta) /(\tau+1), k>k_{0}(\varepsilon, \delta),
$$

a non-principal character $\chi(n)(\bmod k)$ so that

$$
|L(s, \chi)|<1+\varepsilon .
$$

If $k$ is an odd prime, the above theorem gives the result of ANKENY and Chowla (cf. [1], p. 487). 
In the following we denote a character by $\chi^{*}$, when it is a primitive character and we especially want to lay stress on this fact. In this paper we restrict ourselves to the case $k=p^{u}$ ( $p$ is a prime). In this special case we are able to reach a slightly deeper result than the one of theorem 4 . We prove

Theorem 5. Let $\varepsilon$ and $\delta$ be defined in the same way as in theorem 4. If the extended Riemann hypothesis is true, there exists for every given pair $s$ and $p^{u}$, where

$$
s \geqq\left\{\begin{array}{l}
(1+\delta) / 2 \text { if } p>2 \text { and } u \geqq u_{0}=u_{0}(\varepsilon, \delta, p), \\
(2+\delta) / 3 \text { if } p=2 \text { and } u \geqq u_{0}=u_{0}(\varepsilon, \delta),
\end{array}\right.
$$

an odd primitive character $\chi^{*}\left(\bmod p^{u}\right)$, which satisfies the inequality (1).

As a consequence of this we get

Theorem 6. If the conditions of theorem 5 are satisfied, then there exist for every given pair $s$ and $p^{u}$ at least $u-u_{0}+1$ odd characters $\chi\left(\bmod p^{u}\right)$, which satisfy the inequality (1).

3. Consider theorem 3. It is known that there exists for each character $\chi(\bmod m)$ a unique character $\chi^{*}(\bmod f)$ equivalent to $\chi$, where $f=f(\chi)$ is the conductor of $\chi$. We denote by $S$ the set of all these characters $\chi^{*}$ $(\bmod f(\chi))$. Obviously $S$ is also the set of all the primitive characters each of which is equivalent to a character $(\bmod m)$. We need the following lemmas:

Lemma 1. If $S^{\prime}$ is the set $S$ excluding the principal character $\chi_{0}$, we have

$$
h=(2 \pi)^{-\frac{1}{2} \varphi(m)} R^{-1} w \sqrt{|d|} \prod_{\chi \in S^{\prime}} L(1, \chi)
$$

(cf. [4], p. 402).

Lemma 2. Let $\chi\left(\neq \chi_{0}\right)$ be a character $(\bmod m)$, which has the conductor $f$. Then

$$
L\left(1, \chi^{*}\right)=L(1, \chi) \prod_{p \mid m}\left(1-\chi^{*}(p) / p\right)^{-1},
$$

where $\chi^{*}$ is the corresponding primitive character $(\bmod f)$ and $p$ runs through the prime factors of $m$ (cf. [7], p. 127).

Lemma 3. $\omega(m)=O(\log m / \log \log m)$ (cf. [8], p. 108).

Lemma 4. Let $N(k)$ denote the set of the characters $(\bmod k)$. Then

$$
c(\varepsilon) m^{-\varepsilon}<\left|\prod_{\chi \in N} L(1, \chi)\right|<\exp (c(\log \log m+\omega(m)))
$$

where $N^{\prime}$ is the set $N(m)$ excluding $\chi_{0}$ (cf. [8], p. 110). 
A. I. 433

Lemma 5. If $m_{\boldsymbol{t}}$ is the greatest divisor of $m$ prime to $t$, then

$$
\sum_{\chi \in S} \chi(t)=\sum_{\chi \in N\left(m_{t}\right)} \chi(t) .
$$

The proof of this lemma is analogous to the proof of the corresponding result of lemma 7 in [6].

We write, by lemma 2,

$$
\prod_{\chi \in S^{\prime}} L(1, \chi)=\prod_{1} \prod_{2} \prod_{3}
$$

where

$$
\begin{gathered}
\prod_{1}=\prod_{\chi \in N^{\prime}} L(1, \chi), \quad \prod_{2}=\prod_{\chi \in S} \prod_{p \mid m}(1-\chi(p) / p)^{-1}, \\
\prod_{3}=\prod_{p \mid m}\left(1-p^{-1}\right) .
\end{gathered}
$$

We get an estimation for the product $\prod_{1}$ from lemma 4 . Consider the product $\prod_{2}$ (cf. [6], p. 26). It can be expressed in the form

$$
\prod_{1}=\exp \left(\sum_{\chi \in S} \sum_{p \mid m} \sum_{j=1}^{\infty} \chi\left(p^{j}\right) j^{-1} p^{-j}\right) .
$$

Further we have, by lemma 5,

$$
\sum_{\chi \in S} \chi\left(p^{j}\right)=\left\{\begin{array}{c}
\varphi\left(m_{p}\right) \text { if } p^{j} \equiv 1\left(\bmod m_{p}\right), \\
0 \text { otherwise }
\end{array}\right.
$$

where $m_{p}$ denotes the greatest divisor of $m$ prime to $p$. Let $\sigma$ denote the least positive exponent so that

$$
p^{\sigma} \equiv 1\left(\bmod m_{p}\right) .
$$

Now if

$$
p^{v} \equiv 1\left(\bmod m_{p}\right),
$$

then $\sigma \mid v$ and, on the other hand, if $\sigma \mid v$ then (3) holds. Since we can write

$$
\sum_{j=1}^{\infty}\left(p^{-\sigma}\right)^{j}=\left(p^{\sigma}-1\right)^{-1} \leqq m_{p}^{-1},
$$

we have

$$
\sum_{\chi \in S} \sum_{p \mid m} \sum_{j=1}^{\infty} \chi\left(p^{j}\right) j^{-1} p^{-j}=O\left(\sum_{p \mid m} \varphi\left(m_{p}\right) / m_{p}\right)=O(\omega(m)) .
$$

Hence

$$
e^{-c \omega(m)}<\prod_{2}<e^{c \omega(m)}
$$


For $\prod_{3}$ we finally get

$$
2^{-\omega(m)}<\prod_{3}<\omega(m) .
$$

The above results yield, by lemma 3 ,

$$
c(\varepsilon) m^{-\varepsilon}<\left|\prod_{\chi \in S^{\prime}} L(1, \chi)\right|<\exp (c(\log \log m+\omega(m))),
$$

which proves theorem 3. Here $c(\varepsilon)$ and $c$ are not necessarily the same in lemma 4 and in (4).

4. Consider now theorem 5. If $s$ satisfies the conditions (2) and the extended Riemann hypothesis is true, we can write (cf. [6], p. 35)

$$
\prod_{\chi \in Q\left(p^{u}\right)} L(s, \chi)=\exp \left(\varphi\left(p^{u}\right) \psi\left(p^{u}\right)\right)
$$

where $Q\left(p^{u}\right)$ is the set of the odd characters $\chi\left(\bmod p^{u}\right)$, and $\psi\left(p^{u}\right) \rightarrow 0$, when $p^{u}$ tends to infinity. Because $Q\left(p^{u-1}\right) \subset Q\left(p^{u}\right)$, we can decide that

$$
T=: T\left(p^{u}\right)=Q\left(p^{u}\right)-Q\left(p^{u-1}\right)
$$

is the set of the odd primitive characters $\chi^{*}\left(\bmod p^{u}\right)$. Further

$$
\begin{aligned}
\prod_{\chi \in T} L(s, \chi) & =\exp \left(\varphi\left(p^{u}\right) \psi\left(p^{u}\right)-\varphi\left(p^{u-1}\right) \psi\left(p^{u-1}\right)\right) \\
& =\exp \left(\varphi\left(p^{u-1}\right) \psi_{1}\left(p^{u}\right)\right)
\end{aligned}
$$

where

$$
\psi_{\mathbf{1}}\left(p^{u}\right)=p \psi\left(p^{u}\right)-\psi\left(p^{u-1}\right) .
$$

If $p$ is fixed and $u \rightarrow \infty$, then $\psi_{1}\left(p^{u}\right) \rightarrow 0$. Let $g$ denote the number of the odd primitive characters $\left(\bmod p^{u}\right)$. We find that

$$
g=\left\{\begin{array}{l}
1 / 2\left(\varphi\left(p^{u}\right)-\varphi\left(p^{u-1}\right)\right) \text { if } u \geqq 2, \\
1 / 2(p-1) \text { if } u=1 .
\end{array}\right.
$$

In both cases $g \geqq 1 / 2 \varphi\left(p^{u-1}\right)$, and we can see, by (5), that theorem 5 is true.

Because $T\left(p^{j}\right) \cap T\left(p^{j-1}\right)=\Phi(u \geqq j \geqq 2)$ and

$$
\bigcup_{j=u_{0}}^{u} T\left(p^{j}\right) \subset Q\left(p^{u}\right),
$$

we can determine that there exist at least $u-u_{0}+1$ odd characters $\left(\bmod p^{u}\right)$ satisfying the inequality (1). This yields theorem 6.

University of Turku

Turku, Finland 


\section{References}

[1] Ankeny, N. and Chowla, S.: The class number of the cyclotomic field. - Canad. J. Math. 3 (1951), 486-494.

[2] Brauer, R.: On the zeta-functions of algebraic number fields. - Am. J. Math. Vol. 69 No 2 (1947), 243-250.

[3] - 》- On the zeta-funktions of algebraic number fields II. - Am. J. Math. Vol. 72 No 4 (1950), 739-746.

[4] Hasse, H.: Vorlesungen über Zahlentheorie. - 2. Aufl., Springer-Verlag, BerlinGöttingen-Heidelberg-New York (1964).

[5] -»- Zahlenth orie. - 2. Aufl., Akademie-Verlag, Berlin (1963).

[6] Lepistö, T.: On the first factor of the class number of the cyclotomic field and Dirichlet's L-functions. - Ann. Acad. Sci. Fenn. A I 387 (1966).

[7] Prachar, K.: Primzahlverteilung. - Springer-Verlag, Berlin-Göttingen-Heidelberg (1957).

[8] Tatuzawa, T.: On the product of $\mathrm{L}(1, \chi)$. - Nagoya Math. J. 5 (1953), 105-111.

[9] Виноградов, А. И.: О числе классов илеалов и группе класссв дивизоров. - Изв. Акад. Наук СССР, Сер. Мат. 27 (1963), 561-576. 